\title{
Schizophreniform Disorder after Onset of Menopause
}

\author{
Shahzaib Khan1, Melody Dankha² \\ ${ }^{1}$ Ross University School of Medicine, Bridgetown, Barbados \\ ${ }^{2}$ Saint Joseph Mercy Oakland, Pontiac, Michigan \\ Email: shahzaibkhan@mail.rossmed.edu
}

How to cite this paper: Khan, S. and Dankha, M. (2021) Schizophreniform Disorder after Onset of Menopause. Open Journal of Psychiatry, 11, 215-218.

https://doi.org/10.4236/ojpsych.2021.11401

Received: July 10, 2021

Accepted: September 3, 2021

Published: September 6, 2021

Copyright ( 2021 by author(s) and Scientific Research Publishing Inc. This work is licensed under the Creative Commons Attribution International License (CC BY 4.0).

http://creativecommons.org/licenses/by/4.0/

\begin{abstract}
Schizophrenia is one of the psychotic disorders that is a huge concern with prevalence increasing every year. In this case report, we are discussing the case of a 49-year-old female with no prior history of psychiatric illness and a recent diagnosis of menopause, presenting with signs and symptoms of schizophreniform disorder and menopause. After undergoing treatment with estrogen and risperidone, she experienced complete resolution of her delusions and hallucinations.
\end{abstract}

\section{Keywords}

Psychiatric Disorder, Schizophrenia, Schizophreniform Disorder, Delusions, Hallucinations, Menopause

\section{Introduction}

Schizophreniform disorder is diagnosed under the same criteria as schizophrenia according to the DSM 5. To diagnose schizophreniform disorder, psychiatric symptoms must be present for one month to no more than six months and all conditions for schizophrenia must be met. Several studies have suggested dopamine to play a major role in schizophrenic psychosis [1]. One study identified a surge in dopamine storage at the synaptic level in schizophrenic patients [2]. Decreased glutamate levels have also been implicated in the pathophysiology of schizophrenia although further research needs to be done. Currently, research shows that schizophrenic patients have abnormal glutamate receptor localization leading to suboptimal levels in the body [3] [4]. Drug use such as ketamine and PCP have also induced delusions, hallucinations and flat affect due to NMDA antagonism [5].

Menopause is a crucial hormonal transition event in a woman's lifetime. The 
decreasing levels of estrogen and progesterone affect multiple systems in the body including neurotransmitters such as dopamine, acetylcholine and serotonin. Gonadocorticoids have been shown to facilitate the upregulation of dopamine [6]. Estrogen has been proven to strongly modulate NMDA receptors including their neurotransmitter responsiveness, localization and mRNA expression [7] [8].

There are only a few reported cases of schizophrenia diagnosed post menopause, let alone schizophreniform disorder. However, schizophrenic patients have reported exacerbation of their psychosis during the transition from perimenopause to menopause [9].

\section{Case Presentation}

A 49-year-old female was brought in by police for sexual assault work-up. She told the police that three men had forced themselves into the room and sexually assaulted her as well as her 9-year-old daughter. She immediately called the police and upon further questioning, she told them that one of the intruders was her ex-boyfriend. Further inspection showed no forced signs of entry. Her daughter, who was also in the room with her, denied all events. She and her daughter were brought into the hospital for a sexual assault workup which yielded negative results. The patient told the ER nurse that two men had been watching her house every day for the past two weeks, but she did not feel the need to report the incident to the police. Her previous medical history is significant for menopause diagnosed three weeks prior. She has had hot flashes and reported irritable mood. Her lab workup did not show any signs of infection. Urine drug screen was negative.

The patient has no comorbidities. Her brother has a history of depression and she does not keep in touch with her parents. She denies any recent psychological or physical stressor. She denies taking medications or any other recreational drugs. There is no past surgical or psychiatric history. She is a devout Christian and goes to church every Sunday. She works as part of the housekeeping staff at a hotel. On the weekends, she spends time with her best friend and visits her brother sometimes.

Upon questioning the daughter, it was discovered that the patient had been having psychotic symptoms for a month prior to the current event. The patient had repeatedly told the daughter that she was fearful of something bad happening to them and had multiple sleepless nights during this time. She had called her ex-boyfriend multiple times to tell him to stop spying on her and had also started writing, "why are you sending these demons after me, Jesus?" on the walls of her room.

She was admitted to the psychiatric ward for treatment. Her psychiatrist started her on Risperidone and Estrogen. After a week of treatment, she felt better and denied hallucinations. She was discharged on the tenth day of admission with scheduled visits to an outpatient psychiatrist as her discharge plan. 


\section{Discussion}

Schizophreniform disorder is a debilitating psychiatric disorder, affecting many individuals globally. Female schizophrenic patients have a more preferable clinical picture with respect to treatment and tolerance of side effects of different antipsychotic medications [10]. After menopause, however, antipsychotic medications have a declining response, with reported worsening of symptoms in some patients [9] [11]. One study suggests that the efficacy to antipsychotic medications is inversely proportional to the amount of time passed after onset of menopause [11].

This patient was treated with Estrogen for autonomic symptoms pertaining to menopause. She was also started on Risperidone for schizophrenic symptoms. It is unclear whether those medications worked concurrently to treat her psychotic symptoms since no concrete association has been made in research studies. There is evidence that suggests that estrogen therapy as well as raloxifene exceeded expectations to control symptoms in female schizophrenic patients when compared to control groups [12] [13].

This case highlights the gravity of how a major transition, such as menopause, can alter physiology in such drastic ways that it may give rise to a new illness, in this case, schizophreniform disorder. Further research needs to be carried out on the neurotransmitters involved and how to better manage the symptoms of schizophrenia in postmenopausal women.

\section{Conclusions}

Schizophrenia continues to be an enigma in the medical field. Women undergoing menopause experience innumerable hormonal changes which may exacerbate pre-existing schizophrenic psychosis or cause a previously silent disorder to abruptly become clinical. Menopause may be acting as the insult needed to cause a clinically silent disease to suddenly emerge in a pseudo recessive pattern.

Schizophrenia along with its subtypes such as schizophreniform disorder prompts the call for proper clinical management so that it is not a burden on the patient's health and on the financial as well as the social aspect of the health care system. This case highlights the importance of proper history taking and the need for extensive research to be done on how a major transition, such as menopause, could possibly alter the course of a disease which can furthermore give us insight into creating an updated treatment plan.

\section{Conflicts of Interest}

The authors declare no conflicts of interest regarding the publication of this paper.

\section{References}

[1] Laruelle, M. and Abi-Dargham, P. (1999) Dopamine as the Wind of the Psychotic Fire: New Evidence from Brain Imaging Studies. Journal of Psychopharmacology, 
13, 358-371. https://doi.org/10.1177/026988119901300405

[2] Howes, O.D., Fusar-Poli, P., Bloomfield, M., Selvaraj, S. and McGuire, P. (2012) From the Prodrome to Chronic Schizophrenia: The Neurobiology Underlying Psychotic Symptoms and Cognitive Impairments. Current Pharmaceutical Design, 18, 459-465. https://doi.org/10.2174/138161212799316217

[3] Hammond, J., Shan, D., Meador-Woodruff, J. and McCullumsmith, R. (2014) Evidence of Glutamatergic Dysfunction in the Pathophysiology of Schizophrenia. In: Popoli, M., Diamond, D., Sanacora, G., Eds., Synaptic Stress and Pathogenesis of Neuropsychiatric Disorders, Springer, New York, 265-294.

https://doi.org/10.1007/978-1-4939-1056-4_15

[4] Funk, A., Rumbaugh, G., Harotunianc, V., et al. (2009) Decreased Expression of NMDA Receptor-Associated Proteins in Frontal Cortex of Elderly Patients with Schizophrenia. NeuroReport, 20, 1019-1022. https://doi.org/10.1097/WNR.0b013e32832d30d9

[5] Morgan, C.J.A. and Curran, H.V. (2006) Acute and Chronic Effects of Ketamine upon Human Memory: A Review. Psychopharmacology, 188, 408-424. https://doi.org/10.1007/s00213-006-0572-3

[6] Rey, C.D., Lipps, J. and Shansky, R.M. (2014) Dopamine D1 Receptor Activation Rescues Extinction Impairments in Low-Estrogen Female Rats and Induces Cortical Layer-Specific Activation Changes in Prefrontal-Amygdala Circuits. Neuropsychopharmacology, 39, 1282-1289. https://doi.org/10.1038/npp.2013.338

[7] Smith, S.S. and Woolley, C.S. (2004) Cellular and Molecular Effects of Steroid Hormones on CNS Excitability. Cleve. Journal of Clinical Medicine, 71, S4-S10.

[8] Rudick, C.N. and Woolley, C.S. (2001) Estrogen Regulates Functional Inhibition of Hippocampal CA1 Pyramidal Cells in the Adult Female Rat. Journal of Neuroscience, 21, 6532-6543. https://doi.org/10.1523/JNEUROSCI.21-17-06532.2001

[9] Sajatovic, M., Hatters, F.S., Schuermeyer, I.N., et al. (2006) Menopause Knowledge and Subjective Experience among Peri- and Postmenopausal Women with Bipolar Disorder, Schizophrenia and Major Depression. The Journal of Nervous and Mental Disease, 194, 173-178. https://doi.org/10.1097/01.nmd.0000202479.00623.86

[10] Ochoa, S., Usall, J., Cobo, J., Labad, X. and Kulkarni, J. (2012) Gender Differences in Schizophrenia and First-Episode Psychosis: A Comprehensive Literature Review. Schizophrenia Research and Treatment, 2012, Article ID: 916198. https://doi.org/10.1155/2012/916198

[11] González-Rodríguez, A., Catalán, R., Penadés, R., Ruiz Cortés, V., Torra, M., Seeman, M.V. and Bernardo, M. (2016) Antipsychotic Response Worsens with Postmenopausal Duration in Women with Schizophrenia. Journal of Clinical Psychopharmacology, 36, 580-587. https://doi.org/10.1097/JCP.0000000000000571

[12] De Boer, J., Prikken, M., Lei, W.U., et al. (2018) The Effect of Raloxifene Augmentation in Men and Women with a Schizophrenia Spectrum Disorder: A Systematic Review and Meta-Analysis. NPJ Schizophrenia, 4, Article No. 1. https://doi.org/10.1038/s41537-017-0043-3

[13] Zhu, X.M., Zheng, W., Li, X.H., et al. (2018) Adjunctive Raloxifene for Postmenopausal Women with Schizophrenia: A Meta-Analysis of Randomized, Double-Blind, Placebo-Controlled Trials. Schizophrenia Research, 197, 288-293.

https://doi.org/10.1016/j.schres.2018.01.017 